\title{
Sciendo
}

\section{Improving Mental Wellbeing in Organizations with Targeted Psychosocial Interventions}

\author{
Dean Lipovac \\ InnoRenew CoE, Izola, Slovenia \\ Andrej Marušič Institute, University of Primorska, Koper, Slovenia \\ László Hajdu \\ InnoRenew CoE, Izola, Slovenia \\ FAMNIT, University of Primorska, Koper, Slovenia \\ Institute of Informatics, University of Szeged, Szeged, Hungary \\ Sølvi Wie \\ Norwegian University of Life Sciences, Ås, Norway \\ Anders Q. Nyrud \\ Norwegian University of Life Sciences, Ås, Norway
}

\begin{abstract}
Background: Mental wellbeing of employees is crucial for successful organizations. Psychosocial interventions that target highly contagious individuals (i.e., individuals that can 'transmit' their wellbeing to others) could efficiently improve overall wellbeing in the workplace. Objectives: Using the magnitudes of effects observed in existing studies on psychosocial interventions and the contagion of mental wellbeing, we aimed to examine how the wellbeing of a group (based on WHO-5 Well-Being Index scores) changes if interventions are provided to highly contagious people instead of randomly selected individuals. Methods/Approach: Based on the data on mental wellbeing of 414 nursing home employees, we created a social network that includes individual levels of wellbeing and the strength of the connection between people. Simulation-based influence-maximization was used on the network and interventions were interventions were provided to either contagious or randomly selected individuals. Results: Overall, mental wellbeing of the group increased slightly more when individuals had received a simulated psychosocial intervention in order of contagiousness compared to the cases in which interventions were provided to randomly selected individuals. Conclusions: Selectively targeting highly contagious individuals could be an efficient approach to improving wellbeing in organizations, especially in social contexts, where the contagion of mental wellbeing is likelier.
\end{abstract}

Keywords: mental wellbeing, network science, social contagion, infection model, influence maximization

JEL classification: C6

Paper type: Research article

Received: Jan 31, 2020

Accepted: Jul 6, 2020

Citation: Lipovac, D., Hajdu, L., Wie, S., Nyrud, A. Q. (2020), "Improving Mental Wellbeing in Organizations with Targeted Psychosocial Interventions", Business Systems Research, Vol. 11 , No. 2, pp. 86-98.

DOI: 10.2478/bsrj-2020-0017 


\section{Introduction}

Mental wellbeing of employees is critical for the long-term success of an organization. Poor mental wellbeing in the workplace can lead to undesirable outcomes, including absenteeism, loss of productivity, and increased health insurance costs (Danna et al., 1999). It is not surprising that various attempts have been made to improve mental wellbeing in organizations. These approaches can be grouped in organisational-level and individual-level interventions. The former group strives to improve physical environment (e.g., decrease noise), work time conditions (e.g., pace of work), and organisation conditions (e.g., structure of hierarchy) (Montano et al., 2014). The latter group aims to equip individuals with knowledge and skills to better cope with work conditions (e.g., stress management classes) (LaMontagne et al., 2007).

While both approaches are valuable, they each bring their own set of obstacles. Organizational-level interventions are advantageous in simultaneously addressing the entire group of employees, but they often have little or no effect (Briner \& Reynolds, 1999; Montano et al., 2014). On the other hand, interventions aimed at individuals (particularly cognitive-behavioural programmes) can reliably lead to significant positive changes but are less efficient, as they often need to be administered over several weeks in either small groups or one-on-one (Van der Klink et al., 2001; Richardson \& Rothstein, 2008). Despite their effectiveness, sizeable costs required to provide such interventions to all employees might discourage organizations in offering them.

In such cases, a potentially valuable option is to offer only a limited number of individual-level interventions but in a way that could benefit even individuals that themselves do not receive an intervention. This could be achieved by targeting individuals selected based on their ability to "infect" mental wellbeing of other individuals with their own. The approach thus suggests exploiting the phenomenon of mental wellbeing contagion - the observation that mental wellbeing of a particular individual can influence other individuals (Eisenberg et al., 2013). Considering this, a psychosocial intervention could not only improve wellbeing of a highly contagious individual but also positively affect surrounding persons.

The mechanisms behind the contagion of mental wellbeing are numerous and in complex interaction, among them are social comparisons, collaborative development of negative interpretations of recent events, and spreading of (unpleasant) affective states (i.e., core affect, emotions, and mood) (Eisenberg et al., 2013). As an example, consider how affective states can be involved in the contagion process. Unpleasant emotions, such as anger, fear, or sadness, can be "transmitted" between individuals, because people tend to unconsciously mimic facial expressions, voices, movements, and behaviours that can all influence affective states (Hatfield et al., 1993). Chronic experience of such unpleasant affective states (and the lack of pleasant emotions) could contribute to developing mental disorders (Fredrickson, 2000; Fredrickson, 2001; Fredrickson et al., 2003).

The effects of contagion can expand beyond influencing the wellbeing of other group members and can influence group dynamics as a whole, including the attitudes and behaviours of work teams. It has been shown, for example, that when a trained confederate successfully "infected" experiment participants with pleasant affective states, the cooperation between team members increased and conflict decreased (Barsade, 2002). Clearly, transitory affective states seem likelier to spread between individuals than more stable and enduring states of mental wellbeing. Yet it is important to keep in mind that prolonged subtle effects (e.g., increased sadness) could add up to a substantial overall effect (e.g., symptoms of depression) 
(Fredrickson, 2000). Indeed, several studies have observed that the overall mental wellbeing of an individual is influenced by the mental wellbeing of surrounding people (Fowler \& Christakis, 2008; Rosenquist et al., 201 1; Eisenberg et al., 2013).

These findings imply that the contagion of mental wellbeing could be utilized to increase wellbeing of a larger group of people by targeting only select few individuals. Considerably improving mental wellbeing of the most contagious people could be a more efficient approach to improve overall wellbeing of the entire personnel, when compared to directly but slightly improving wellbeing in each employee (as could be achieved with certain organisational-level interventions).

The first step in this approach is identifying highly contagious people. The contagiousness of individuals depends on many factors, both personal and contextual. Important personal characteristics include contagion ability (e.g., emotional expressiveness) and susceptibility (Clarkson et al., 2020). Contextual factors include, for instance, the nature and amount of time individuals spend together. Supervisors are an obvious example of individuals who might be especially prone to being contagious, as they tend to be important in lives of their subordinates and ordinarily have many social connections (Coenen \& Broekens, 2012; Eisenberg et al., 2013). Providing psychosocial interventions to such highly contagious people might disproportionately improve the mental wellbeing of the surrounding group of people.

Our objective is to examine if, at least in theory, selectively targeting highly contagious individuals with psychosocial interventions can be an efficient solution to improve overall wellbeing of a larger group of people. We will explore this by running social network infection simulations based on empirically derived effect sizes representing real-life effects of psychosocial interventions and the degree of mental wellbeing contagion. The simulation of the infection process can show if the overall wellbeing of the entire group is disproportionately improved when highly contagious people are targeted with psychosocial interventions (compared to randomly selected individuals). We hypothesise that the mental wellbeing of the entire group of people will improve to a larger degree when the simulated intervention is provided in the order of contagiousness (highly contagious individuals receive it first) instead of random order.

\section{Methodology}

The proposed methodology might be valuable in optimizing wellbeing of large groups, by exploring how the mental wellbeing of all people in the group changes in response to providing psychosocial interventions to different individuals. It is important to point out that the extent of potential changes in wellbeing relies heavily on the input parameters (e.g., degree of mental contagion), which could substantially differ between social contexts. Thus, although the approach will be presented through empirical data, we would like to emphasize that the method might be useful and worth exploring in other contexts with different parameters, such as different characteristics of individuals (e.g., age, gender, personality) and the environment (e.g., proximity of other people).

As a first step, we created a social network based on the data collected on nursing home employees. From the existing empirical studies, we then selected the effect size of the most effective individual-level intervention in organizations to serve as the effect size of the hypothetical psychosocial intervention provided in our simulation. Similarly, we selected an empirically derived effect size representing the degree of mental wellbeing contagion. These data were used by a simulation model that aimed to improve the overall wellbeing of the entire group of employees by providing a psychosocial intervention to the most contagious people that had been selected 
based on several parameters. The overall wellbeing score resulting from this simulation was compared with the score from the simulation in which individuals receiving the psychosocial intervention were selected at random. Each step is presented in more detail below.

\section{Data collection and transformation}

We collected the data on 414 employees from 14 nursing homes in Norway, who completed the survey capturing demographic data, work-related information (e.g., occupation, years working, working hours, shift work), and levels of wellbeing.

We used only the data from participants that had completed the questionnaire assessing wellbeing and who, based on the assumptions of our simulation model, had at least one social connection. These conditions were met by 278 people (268 women), with the mean age of 46.94 years (from 19 to $70 ; S D=11.375$ ). Most persons were employed as nurses and auxiliary nurses (235), followed by other healthcare workers (24), supporting staff (14), and managers (3).

Wellbeing was assessed with the WHO-5 questionnaire (WHO, 1998) that asks five questions pertaining to the subject's last two weeks (e.g. "I have felt cheerful and in good spirits."). Subjects answered each question on a six-point Likert-type scale $10=$ "At no time", 5 = "All of the time"). The results for one item of the questionnaire ("I have felt calm and relaxed.") were missing in our data, so we calculated the final score from the remaining four items. We summed the values of responses to obtain the raw score and then rescaled it to obtain a percentage score ranging between 0 and 100 (larger number represents higher levels of wellbeing). In our sample, the mean percentage score on WHO-5 was 68.09 (SD = 17.17). Percentage score is recommended when changes in wellbeing are monitored and this score is used in the results and discussion section. For the purposes of the simulation, however, the score was first divided by 100 to obtain values between 0 and 1 and then reversed, so the values closer to 0 represent higher levels of wellbeing. This reversal was necessary due to the nature of the infection model simulation, which is described in the following sections.

\section{Effect sizes used in the simulation}

Individual-level intervention effect size: A meta-analysis of various individual-level interventions in organizations reported that cognitive-behavioural programmes produced the largest average effect size (Cohen's d (standardised difference between two means expressed in SD) = 1.1154) for a combined group of mental wellbeing outcomes that included measures of stress, anxiety, mental health, and work-related outcomes (e.g., work satisfaction, motivation, perceived control) (Richardson \& Rothstein, 2008). This effect size was incorporated in our model; on average, every employee targeted by an intervention had their wellbeing score increased by 1.1554 multiplied by the standard deviation of the WHO-5 percentage score rescaled between 0 and 1 (in our case, SD $=0.172$ ). To approximate varying effects expected in real-life, the effect size of the intervention provided to each employee varied according to the normal distribution with the mean of 1.1554 and standard deviation set arbitrarily at 0.10 .

Mental wellbeing contagion effect size: Among the identified studies examining mental wellbeing contagion (Fowler \& Christakis, 2008; Rosenquist et al., 2011; Eisenberg et al., 2013), we selected a study from Eisenberg et al. (2013) that was especially careful in controlling several sources of bias and, correspondingly, arrived at a lower estimate of the mental contagion effects compared to other studies $(\beta=$ $0.053,95 \% \mathrm{Cl}=[0,0.12])$. Although this effect size is based on specific anxiety items 
from the K-6 instrument assessing general psychological distress (Kessler et al., 2003), the captured construct has been shown to have a considerable overlap with the construct tapped by WHO-5 (e.g., Downs et al. 2017), on which we base our simulation. For our simulation, the selected effect size indicates that the wellbeing score of a neighbour in a social network will increase for 0.053 multiplied by the standard deviation of the WHO-5 percentage score rescaled between 0 and 1 (SD = 0.172). To allow for varying degrees of contagion based on the strength of social connections (e.g., amount of time spent together), we instructed our simulation model to select a value from the $95 \%$ confidence interval $[0,0.12]$ of the abovementioned effect size, where the stronger social connection received a higher value. The resulting values that are used in the simulation thus lie on the interval between 0 (i.e., $0.172 * 0$ ) and 0.021 (i.e., $0.172 * 0.12$ ).

\section{Network modelling}

General model: To define the network formally, let $G(V, E)$ be the network where $V$ is the set of the nodes (in our case the set of employees) and $E$ describes the set of edges (the connections between the nodes). Let $0 \leq p_{v_{1}, v_{2}} \leq 1$ be the edge probability between $v_{1}$ and $v_{2}$, where $v_{1}, v_{2} \in V$. This probability represents the connection strength between two nodes; 1 signifies the strongest connection and 0 indicates there is no connection. In addition, let us define the properties of a node:

- Let $s_{v}$ be an initial probability, representing the reversed and rescaled WHO-5 percentage score of the node $v$

- let us call $s_{v}^{\text {intervention }}$ the intervention probability of the node $v$, which describes the reversed WHO-5 score of the employee after the intervention, therefore $s_{v}^{\text {intervention }} \leq s_{v}$.

- $\quad w_{v_{1}}, w_{v_{2}}, w_{v_{3}} \ldots w_{v_{n}}$ a list of real-life based properties of the $v$ node

In case of both nodes and edges, the initial probability comes from a real-life based property (i.e., our data) of the node (i.e., employee). In the following section, we describe the network created from the collected data.

Model from the collected data: To give an instance of a general model, we used the collected data from the nursing homes. Since we did not have information about the real connections between the employees, we created the connection structure based on similarities of different individuals. In the network, every employee is represented by a node and the connections (i.e., edges) between them were arbitrarily assigned if:

- They were employed at the same nursing home

- They had the same occupation (e.g., nurse)

- The age difference between them was not greater than 20 years.

The strength of the connection was computed based on properties of the corresponding employees, resulting from the sum of the following properties:

- Age difference: Difference in age of the corresponding employees, where lower age difference increases the connection strength, scaled between 0 and 0.33

- Matching work shifts: The probability of employees meeting during work due to similar work schedules, where matching shifts increase the connection strength, scaled between 0 and 0.33

- Weekly working hours difference: The probability of employees meeting during work due to similar working hours, where a similar number of working hours increases the connection strength, scaled between 0 and 0.33 
The final edge weight is the sum of the scaled values, so a number between 0 and 1 , multiplied by a random number between 0 and 0.021 , which represents the extent of mental wellbeing contagion (the process of arriving at this value is described in a previous section). (It is important to point out that the model is flexible enough to be used with different connection strengths; to compute the edge probabilities based on different edge attributes, work from Bóta et al. (2014) provides a good example.)

The resulting network had 289 nodes and 731 edges. A sample of the network is presented in Figure 1. Nodes, representing employees, are coloured based on the rescaled and reversed $\mathrm{WHO}-5$ scores; the width of the edges increases with the edge weight (i.e., probability of the mental wellbeing contagion).

Figure 1

A Sample of the Network

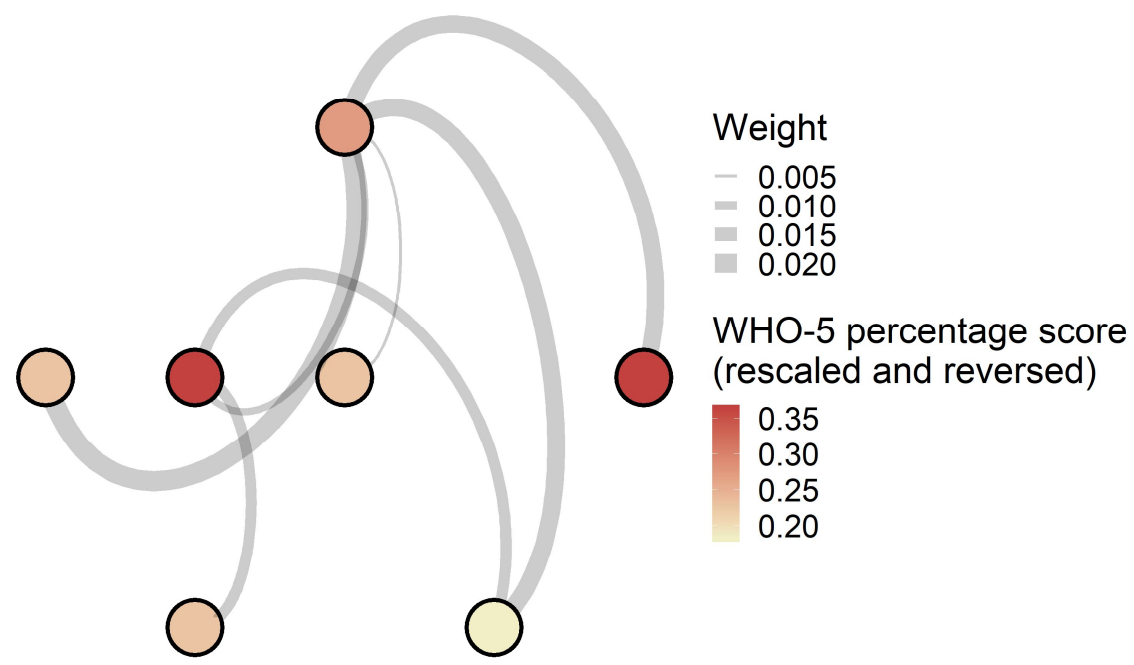

Source: Authors' work

\section{Infection model and optimization}

The basic idea of infection models is to simulate the spread of a virus, information, or any other entity on a social network. In our case, this entity is human mental wellbeing (reversed score), as it can be contagious in a similar way as other effects on the network (e.g., Eisenberg et al., 2013). The basic concept of the problem was proposed by Domingos \& Richardson (2001) and by Granovetter (1978), where the idea and the objective of the research was to improve the efficiency of viral marketing. The exact mathematical description and theoretical background of a problem was introduced by Kempe et al. $(2003,2005)$.

To represent employee connections and the spreading of (reversed) wellbeing levels in the workplace, we used an extended Generalized Independent Cascade model (Bóta et. al., 2013), where initial probabilities on the nodes are also defined. Chen et al. (2010) proved that the exact computation of the node probabilities is P\#complete, therefore, mostly heuristics are used. However, with simulation, any precision level can be reached (Kempe et al., 2003).

If the previously defined network is given with all of its properties, let $\boldsymbol{f}_{v}$ be the final reversed wellbeing score of the node $\boldsymbol{v}$ after the simulation, and value $\boldsymbol{\sigma}(\boldsymbol{V})$ the sum of the final infection for each node, which was computed by the Complete Simulation (Bóta et al., 2013). The difference between the mentioned models and our model is 
that in our case every single node can become an infector in the first step of the algorithm. The method takes the following inputs:

- $\boldsymbol{G}(\boldsymbol{V}, \boldsymbol{E})$ previously defined social network

- $\quad \boldsymbol{N} \subset \boldsymbol{G}(\boldsymbol{V})$ employee set without a psychosocial intervention

- $\quad \boldsymbol{I} \subset \boldsymbol{G}(\boldsymbol{V})$ employee set with a psychosocial intervention

- $\boldsymbol{k}$ sample size

It is important to note that $\boldsymbol{N} \cap \boldsymbol{I}=\emptyset$ and $\boldsymbol{N} \cup \boldsymbol{I}=\boldsymbol{G}(\boldsymbol{V})$. The pseudocode of the simulation is presented in Algorithm 1.

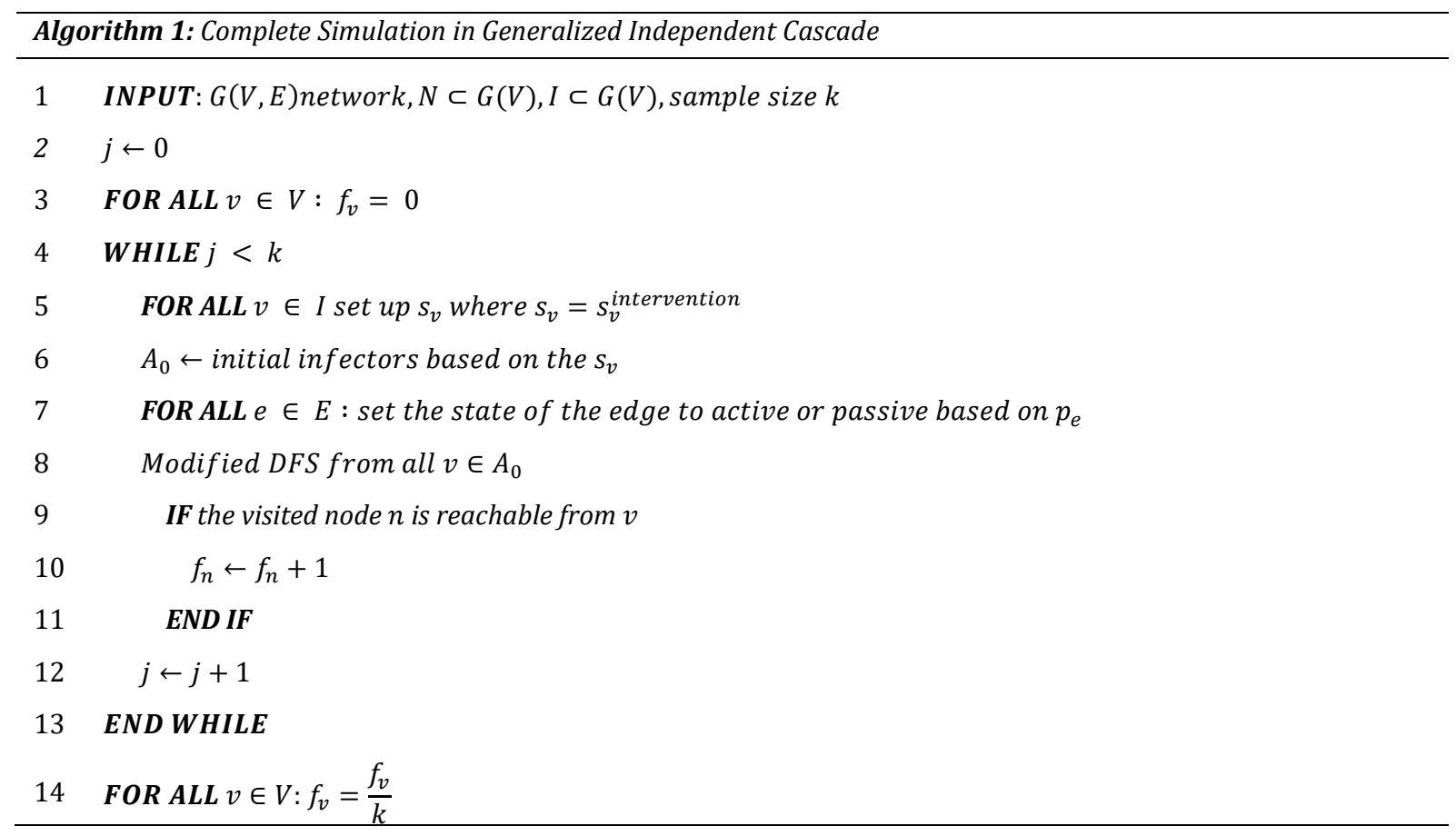

The algorithm generates $k$ different instances of the network by deleting and keeping edges and creating infectious and non-infectious employees, using the edge and the node probabilities. The simulation is used to compute the final reversed wellbeing score of one possible scenario, where $I$ and $N$ are given; that is, with one possible employee set receiving the intervention. First, we run a reference simulation to get the final infection in case if $I=\emptyset$ (no intervention). The optimization environment computes the possible intervention scenarios and maximizes the overall wellbeing by minimizing the scores of the nodes (i.e., reversed wellbeing score) with the set of employees receiving the intervention. Figure 2 shows the basic frame of the system with example values. 
Figure 2

Optimization Environment

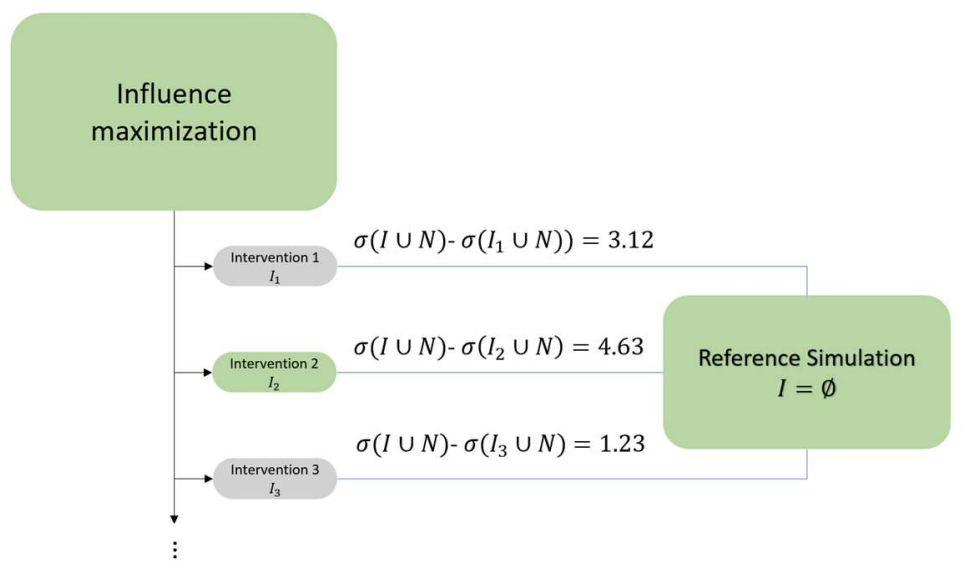

Source: Authors' work

In this case, the intervention 2 was chosen, since the $I_{2}$ set of employees receiving intervention reached the maximal reduction in reversed wellbeing score in the model. After the intervention, the model will decrease the score of each employee in the $I$ set and their local neighbourhood, since the employee receiving intervention will now have a lower probability to spread its "reversed wellbeing". A similar model, where the negative spread was considered, was published by Tóth (2016). To optimize the influence of the intervention in the social network, we used the infection maximization.

In the infection maximization problem, the main objective is to maximize the spread with an initial infector set. The original infection maximization problem was published by Kempe et al. (2003), where they proved the NP-hardness of the problem. Due to the hardness of the problem, we used a heuristic to maximize the intervention effect on the network. The most efficient and widely used method with a guaranteed solution is the greedy method. In the same paper, Kempe et al. (2003) proved that the greedy method gives at least $63 \%$ of the optimum. In our case, the greedy method maximizes the difference between the reference simulation and the actual solution; therefore, to use the greedy method for our research problem, we had to change certain parts of it. The pseudocode of the proposed method for the optimization is the following:

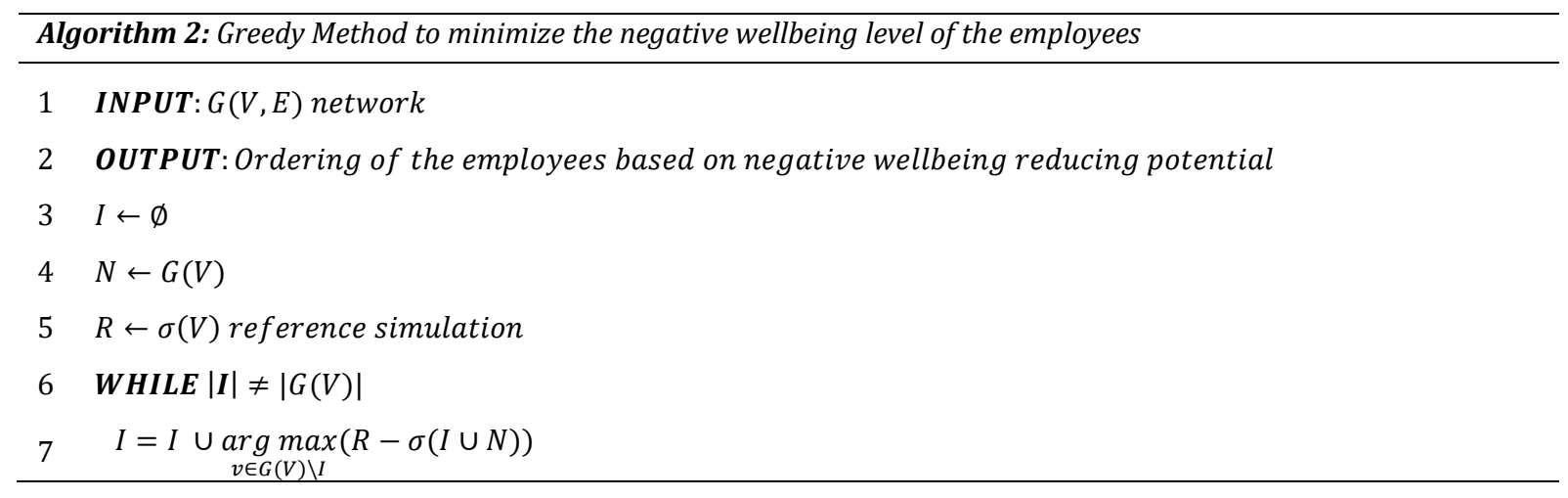

The greedy algorithm increases the size of the $I$ by one in every iteration, by selecting the employee that decreases the global reversed wellbeing score the most. To show the optimal number of the employees receiving the intervention, it is possible to find a threshold where the global negative wellbeing will stop decreasing significantly. 


\section{Results and discussion}

We compared the changes in wellbeing of the entire group of people between two instances: when simulated interventions were provided either in order of contagiousness or in random order. Figure 3 displays the mean increase in $\mathrm{WHO}-5$ percentage score per person after the hypothetical intervention was provided to different number of individuals in the group (compared to the reference point scores without the intervention). The figure separates the scores based on the order in which the intervention was administered; in one case, the order of individuals provided with the intervention was random, in the other, the intervention was first administered to highly contagious people (i.e., people who, after receiving the intervention, made the largest positive impact on the wellbeing of the entire group of people). In both cases, the scores steadily increase until all individuals receive the intervention, where the average increase becomes similar to the effect size of the intervention used in the simulation. When the intervention was administered to highly contagious people first, the increases in scores were generally larger when compared to the scores following random administration of interventions. This represents the effect of contagion: although, on average, the score of each individual increased (i.e., improved) the same after the intervention, some persons were better able to spread that improvement to others, due to their contagiousness.

Figure 3

Mean Increase in WHO-5 Depending on the Order of Intervention Administration

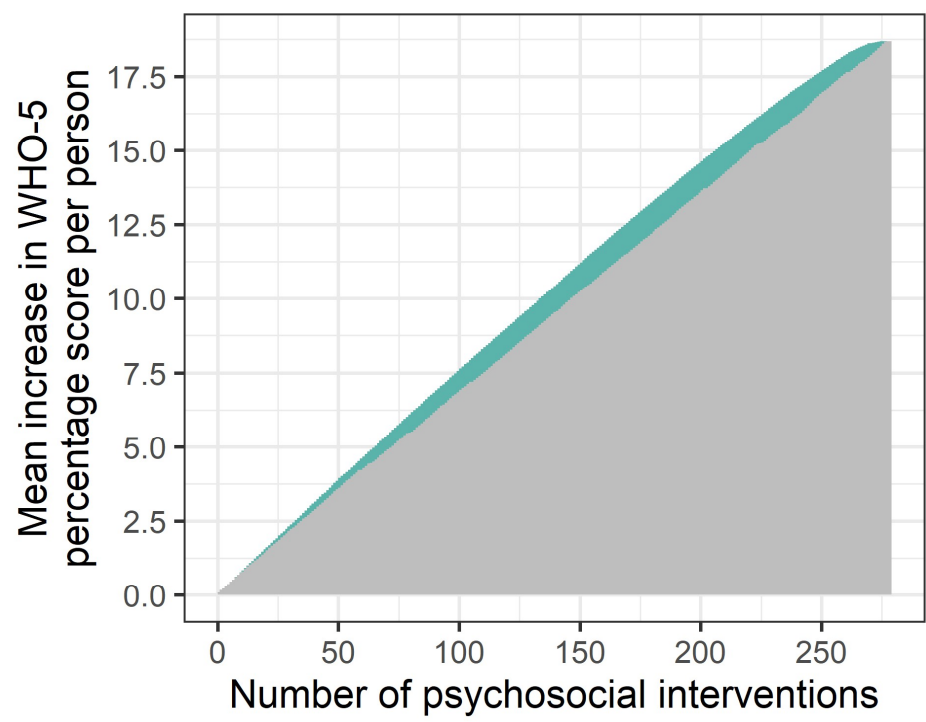

The order of administering the intervention

Random

Highly contagious first

Source: Authors' work

Some of the results from Figure 3 are presented in more detail in Table 1.

Table 1

Comparison of the WHO-5 Percentage Score Mean Increase per Person

\begin{tabular}{cccc}
$\begin{array}{c}\text { Number and } \\
\text { percent of } \\
\text { interventions }\end{array}$ & $\begin{array}{c}\text { Mean increase in } \\
\text { score after random } \\
\text { administrations }\end{array}$ & $\begin{array}{c}\text { Mean increase in } \\
\text { score after targeted } \\
\text { administrations }\end{array}$ & $\begin{array}{c}\text { Difference between } \\
\text { targeted and random }\end{array}$ \\
$10(3.6 \%)$ & 0.74 & 0.83 & 0.09 \\
$20(7.2 \%)$ & 1.43 & 1.61 & 0.18 \\
$50(18.0 \%)$ & 3.55 & 3.94 & 0.38 \\
$100(36.0 \%)$ & 6.85 & 7.64 & 0.79 \\
$200(71.9 \%)$ & 13.58 & 14.64 & 1.06 \\
\hline
\end{tabular}

Source: Authors' work 
The table 1 displays the mean increase in $\mathrm{WHO}-5$ percentage score per subject for selected numbers of interventions provided to the group. For example, after 20 subjects received the simulated intervention, the percentage score in the entire group of subjects increased, on average, by 1.61 per person, when intervention administrations were ordered by contagiousness, which is 0.18 larger than the average increase per person following randomly administered interventions. In this case, selectively targeting contagious individuals is thus responsible for a 0.18 increase in WHO-5 percentage score per person, all else being equal.

The observed differences in mean scores between random and targeted intervention administrations are small. This is not surprising, given that our model was based on a relatively large effect size following an intervention, but only a fraction of that improvement was expected to be transmitted between individuals, due to the small effect size of mental wellbeing contagion that was incorporated in the simulation model. However, in contrast with the intervention effect size that was based on a meta-analysis considering several studies, the contagion effect size was derived from a single study (Eisenberg et al., 2013), due to lack of relevant research.

Despite the robustness of that study, there are reasons to assume that the contagion effect could be larger. As is generally the case, a single result can rarely be a definitive answer on the topic. Indeed, other studies researching mental wellbeing contagion in other contexts have arrived at considerably larger effect sizes (although, admittedly, in those studies the potential for bias was higher) (Fowler \& Christakis, 2008; Rosenquist et al., 2011). Another important aspect is the social context. Our model used an effect size derived from a study examining contagion in college roommates. In different contexts, however, the contagion effect could be larger, as it may depend on various individual and interpersonal factors (Coenen \& Broekens, 2012) Difference in social status is one of the factors increasing the degree of contagion; contagion is likely more pronounced when passing between a higher social status individual and one with a lower status (e.g., between a supervisor and a subordinate) (Coenen \& Broekens, 2012). Presumably, such asymmetries in social status are more common in many hierarchically structured organizations than in relationships between college roommates, on which our contagion effect size was based. If the contagion of mental wellbeing is indeed more pronounced in certain organizations, our simulation model could show that the overall effects of contagion are considerably larger compared to the effects reported in this article. The effects could be particularly pronounced, for example, in highly hierarchical organizations, where supervisors hold especially high status compared to their subordinates (presumably leading to larger contagion effects) while at the same time supervising many employees (i.e., there are numerous recipients of the contagion effects). An organization could identify such potentially highly contagious individuals with a simulation model, assuming appropriate data is available or can be collected. Interventions targeted at such individuals could lead to a relatively large improvement in the wellbeing of the entire group.

\section{Limitations}

The model used in our study could represent real-life more closely if additional data would be considered as a moderator of the contagion effects. Since it was shown that various individual and interpersonal factors may influence the degree of contagion (Coenen \& Broekens, 2012), taking these data into account is crucial. For example, females might be more susceptible to contagion, and contagion might be more pronounced between people with similar attitudes on various topics (e.g., religion, sports, death penalty) (Coenen \& Broekens, 2012). The model could be additionally strengthened after an empirical evaluation (e.g., Tsai et al., 2011). 
Although beyond the scope of this article, it is worth pointing out that providing interventions to a subset of individuals might provide practical hurdles that are challenging to overcome. For example, individuals that are selectively offered a psychosocial intervention might receive the offer negatively (dve to the stigma related to implied issues with mental wellbeing), while individuals not offered the intervention could react negatively as well (due to perceiving the lack of offer as unjust).

\section{Conclusion}

Improving mental wellbeing is a challenging task, especially when attempting to improve wellbeing of a large group of people. Interventions can often only slightly improve the overall wellbeing in the workplace. Organizational-level interventions can address the entire personnel simultaneously, but provide little effect, while interventions targeting individuals provide considerably larger effects, but can require substantial resources in terms of time, effort, and money. Either way, regardless of which intervention type is selected, many individuals will be in need of additional support. Clearly, then, the efficiency of interventions is of interest. One way to increase the efficiency of existing interventions, is to provide them to specific individuals - those who are highly contagious and can 'transmit' their mental wellbeing to other people. In effect, those individuals can make the most of the intervention, as far as the overall wellbeing of a group is concerned.

We have seen, however, that the effects of the mental wellbeing contagion can be relatively small and that singling out individuals, who are selected to receive the intervention, might bring additional challenges. Yet it is important to keep in mind that in different contexts the contagion effects could be larger and that issues stemming from singling out individuals might be well worth the price, considering the subsequent improvement in overall wellbeing of the group. We have shown that, at least in principle, the wellbeing of a group can be more efficiently improved if highly contagious people are targeted with interventions. As this approach could improve the efficiency of psychosocial interventions, leading to improved wellbeing in organizations, it is worth further theoretical and empirical exploration.

\section{Acknowledgments}

The authors gratefully acknowledge the European Commission for funding the InnoRenew CoE project (Grant Agreement 739574) under the Horizon2020 Widespread-Teaming program, the Republic of Slovenia (Investment funding of the Republic of Slovenia and the European Union of the European Regional Development Fund), and the Slovenian Research Agency ARRS for funding infrastructure program $10-0035$.

\section{References}

1. Barsade, S. G. (2002), "The ripple effect: emotional contagion and its influence on group behavior", Administrative Science Quarterly, Vol. 47, No. 4, pp. 644-675.

2. Bóta, A., Krész, M., Pluhár, A. (2013), "Approximations of the generalized cascade model", Acta Cybernetica, Vol. 21, No.1, pp. 37-51.

3. Bóta, A., Krész, M., Pluhár, A. (2014), "The inverse infection problem", in Ganzha, M., Maciaszek, L., Paprzycki, M. (Eds.), 2014 Federated Conference on Computer Science and Information Systems, Annals of Computer Science and Information systems, Vol. 2, 7-10 September, IEEE, Warsaw, pp. 75-84.

4. Briner, R. B., \& Reynolds, S. (1999), "The costs, benefits, and limitations of organizational level stress interventions", Journal of Organizational Behavior, Vol. 20, No. 5, pp. 647-664.

5. Chen, W., Yuan, Y., Zhang, L. (2010), "Scalable influence maximization in social networks under the linear threshold model", in Webb, G. I., Liu, B., Zhang, C., Gunopulos, D., Wu, X. 
(Eds.), 2010 IEEE International Conference on Data Mining, 13-17 December, IEEE, Sydney, pp. 88-97.

6. Clarkson, B. G., Wagstaff, C. R., Arthur, C. A., Thelwell, R. C. (2020), "Leadership and the contagion of affective phenomena: a systematic review and mini meta-analysis", European Journal of Social Psychology, Vol. 50, No.1, pp. 61-80.

7. Coenen, R., Broekens, J. (2012), "Modeling emotional contagion based on experimental evidence for moderating factors", in 11 th International Conference on Autonomous Agents and Multiagent Systems, 4-8 June, IFAAMAS, Valencia, Vol. 3., pp. 4-8.

8. Danna, K., Griffin, R. W. (1999), "Health and well-being in the workplace: a review and synthesis of the literature", Journal of Management, Vol. 25, No.1, pp. 357-384.

9. Domingos, P., Richardson, M. (2001), "Mining the network value of customers", 7th ACM SIGKDD International Conference on Knowledge Discovery And Data Mining, 26-29 August, Association for Computing Machinery, New York, pp. 57-66.

10. Downs, A., Boucher, L. A., Campbell, D. G., Polyakov, A. (2017), "Using the WHO-5 wellbeing index to identify college students at risk for mental health problems", Journal of College Student Development, Vol. 58, No.1, pp. 113-117.

11. Eisenberg, D., Golberstein, E., Whitlock, J. L., Downs, M. F. (2013), "Social contagion of mental health: evidence from college roommates", Health Economics, Vol. 22, No. 8, pp. 965-986.

12. Fowler, J. H., Christakis, N. A. (2008), "Dynamic spread of happiness in a large social network: longitudinal analysis over 20 years in the Framingham Heart Study", BMJ, Vol. 337, a2338.

13. Fredrickson, B. L. (2000), "Cultivating positive emotions to optimize health and well-being", Prevention \& Treatment, Vol. 3, No. 1, 1.

14. Fredrickson, B. L. (2001), "The role of positive emotions in positive psychology: the broadenand-build theory of positive emotions", American Psychologist, Vol. 56, No. 3, pp. 218-226.

15. Fredrickson, B. L., Tugade, M. M., Waugh, C. E., Larkin, G. R (2003), "What good are positive emotions in crises? A prospective study of resilience and emotions following the terrorist attacks on the United States on September 11 th, 2001", Journal of Personality and Social Psychology, Vol. 84, No. 2, pp. 365-376.

16. Granovetter, M. (1978), "Threshold models of collective behaviour", American Journal of Sociology, Vol. 83, No. 6, pp. 1420-1443.

17. Hatfield, E., Cacioppo, J. T., Rapson, R. L. (1993), "Emotional contagion", Current Directions in Psychological Science, Vol. 2, No. 3, pp. 96-100.

18. Kempe, D., Kleinberg, J., Tardos, É. (2003), "Maximizing the spread of influence through a social network", in Domingos, P. (Ed.), 9th ACM SIGKDD International Conference on Knowledge Discovery and Data Mining, 24-27 August, Association for Computing Machinery, New York, pp. 137-146.

19. Kempe, D., Kleinberg, J., Tardos, E. (2005), "Influential nodes in a diffusion model for social networks", in Caires, L., Italiano, G.F., Monteiro, L., Palamidessi, C., Yung, M. (Eds.), 32nd International Colloquium on Automata, Languages and Programming ICALP 2005, 11-15 July, European Association for Theoretical Computer Science Lisbon, pp. 1127-1138.

20. Kessler, R. C., Barker, P. R., Colpe, L. J., Epstein, J. F., Gfroerer, J. C., Hiripi, E., Howes, M. J., Normand, S. L. T., Manderscheid, R. W., Walters, E. E., Zaslavsky, A. M. (2003), "Screening for serious mental illness in the general population", Archives of General Psychiatry, Vol. 60, No. 2, pp. 184-189.

21. LaMontagne, A. D., Keegel, T., Lovie, A. M., Ostry, A., Landsbergis, P. A. (2007), "A systematic review of the job-stress intervention evaluation literature, 1990-2005", International Journal of Occupational and Environmental Health, Vol. 13, No. 3, pp. 268280.

22. Montano, D., Hoven, H., Siegrist, J. (2014), "Effects of organisational-level interventions at work on employees health: a systematic review", BMC Public Health, Vol. 14, No. 1, 135.

23. Richardson, K. M., Rothstein, H. R. (2008), "Effects of occupational stress management intervention programs: a meta-analysis", Journal of Occupational Health Psychology, Vol. 13, No. 1, pp. 69-93.

24. Rosenquist, J. N., Fowler, J. H., Christakis, N.A. (2011), "Social network determinants of depression", Molecular Psychiatry, Vol. 16, No. 3, pp. 273-281. 
25. Tóth, L. (2016), "Greedy heuristics for the generalized independent cascade model", in Fister, I., Brodnik, A. (Eds.), 2016 3rd Student Computer Science Research Conference StuCoSReC, 12 October, University of Primorska Press, Koper, pp. 51-54.

26. Tsai, J., Bowring, E., Marsella, S., Tambe, M. (2011), "Empirical evaluation of computational emotional contagion models", in Vilhjálmsson, H. H., Kopp, S., Marsella, S., Thórisson, K. R., (Eds.), 10th International Conference Intelligent Virtual Agents, 15-17 Septembar, Springer, Berlin, Heidelberg, pp. 384-397.

27. Van der Klink, J. J., Blonk, R. W., Schene, A. H., Van Dijk, F. J. (2001), "The benefits of interventions for work-related stress", American Journal of Public Health, Vol. 91, No. 2, pp. 270-276.

28. WHO. (1998), "Wellbeing measures in primary health care/the depcare project", available at http://www.euro.who.int/_data/assets/pdf_file/0016/130750/E60246.pdf / (30 January 2020)

\section{About the authors}

Dean Lipovac completed a master's degree in applied psychology at the Faculty of Mathematics, Natural Sciences and Information Technologies, University of Primorska (UP), in Koper, Slovenia and is currently a PhD student. He is an assistant researcher at the InnoRenew CoE and Andrej Marušič Institute (UP). His main research interest is the connection between the built environment and human mental health. His work is focused on researching how different materials used in indoor design influence physiological, emotional, and cognitive indicators of well-being. The author can be contacted at dean.lipovac@innorenew.eu

László Hajdu is an assistant researcher at Innorenew CoE, at University of Primorska, Faculty of mathematics, Natural Sciences and information technologies, and PhD Student at the University of Szeged. He completed his Bachelor's and Master's degree in Computer Science at the University of Szeged. His main research interest is network science and graph algorithms. His work is focused mostly on Community Detection, Infections in networks, Banking Data Analysis, and Network Science based optimization methods. The author can be contacted at laszlo.hajdu@innorenew.eu

Sølvi Wie, MSc, is a Ph.D. student in Wood Technology at the Faculty of Environmental Science and Natural Resource Management, Norwegian University of Life Sciences (NMBU). She received MSc in Public Health Science at the Faculty of Landscape and Society, NMBU, with the thesis "Associations between work-related psychosocial risk factors and musculoskeletal complaints in Norwegian aircrew". Her main research interest is the effect of nature, environment, and surroundings on human health; currently she is investigating the relationship between wood in buildings and occupant experience and health. The author can be contacted at solvi.wie@nmbu.no

Anders Q. Nyrud, Dr, scient, is a Professor in Wood Technology at the Faculty of Environmental Science and Natural Resource Management, Norwegian University of Life Sciences (NMBU). Professor Nyrud has a background from research and consulting in the Norwegian wood processing industries. His research focuses on various aspects related to the use of wood, in particular the relationship between raw material properties, industrial production processes, and market requirements for final products and their attributes (i.e., functional, aesthetical and/or psychological attributes). The author can be contacted at anders.qvale.nyrud@nmbu.no 